\title{
Reflets
}

Revue ontaroise d'intervention sociale et communautaire

\section{L’ATTSO : la voix des travailleuses et travailleurs sociaux en Ontario}

\section{Rita Mascherin}

Volume 7, numéro 2, automne 2001

Le travail social en Ontario

URI : https://id.erudit.org/iderudit/026360ar

DOI : https://doi.org/10.7202/026360ar

Aller au sommaire du numéro

Éditeur(s)

Reflets : Revue ontaroise d'intervention sociale et communautaire

ISSN

1203-4576 (imprimé)

1712-8498 (numérique)

Découvrir la revue

Citer cet article

Mascherin, R. (2001). L’ATTSO : la voix des travailleuses et travailleurs sociaux en Ontario. Reflets, 7(2), 132-140. https://doi.org/10.7202/026360ar

Tous droits réservés (C) Reflets : Revue ontaroise d'intervention sociale et communautaire, 2001
Ce document est protégé par la loi sur le droit d'auteur. L'utilisation des services d’Érudit (y compris la reproduction) est assujettie à sa politique d'utilisation que vous pouvez consulter en ligne.

https://apropos.erudit.org/fr/usagers/politique-dutilisation/ 


\section{L'ATTSO : la voix des travailleuses et travailleurs sociaux en Ontario}

par

Rita Mascherin, adjointe de direction, Association des travailleuses et travailleurs sociaux de l'Ontario

En tant que travailleuses et travailleurs sociaux exerçant dans l'une des principales professions de services à la personne, vous connaissez les défis que représente un milieu de travail en évolution rapide. L'Association des travailleuses et travailleurs sociaux de l'Ontario (ATTSO) est la voix des travailleuses et travailleurs sociaux en Ontario.

L'adhésion à l'ATTSO permet l'accès à des services rapides et efficaces:

- une assurance responsabilité professionnelle sans laquelle vous risquez d'encourir d'énormes frais en poursuites judiciaires;

- des ressources et des services pour maintenir vos compétences professionnelles et vous tenir au courant des tendances dans la pratique;

- des services de défense concernant d'importantes questions professionnelles et sociales dans votre domaine de pratique;

- des moyens d'établir des contacts avec d'autres travailleuses et travailleurs sociaux;

- de la documentation, des services et des possibilités d'emploi à jour;

- des informations et des services visant les étudiants en service social. 
L'ATTSO, association bilingue de membres, fait partie de l'Association canadienne des travailleuses et travailleurs sociaux (ACTS) et de la Fédération internationale des travailleurs sociaux (FITS) qui regroupe 76 pays.

L'ATTSO représente activement les intérêts et les préoccupations des travailleuses et travailleurs sociaux:

- en réagissant à l'impact des politiques et programmes gouvernementaux sur la pratique professionnelle;

- en encourageant la reconnaissance du rôle et des compétences du service social;

- en mettant en question les perceptions erronées au sujet de la profession qui ressortent dans les médias, les organismes et la communauté;

- en faisant pression auprès des compagnies d'assurance pour que nos honoraires soient couverts par des régimes de santé privés.

L'ATTSO incarne l'engagement de la profession envers une société civile équitable:

- en se lançant dans l'action sociale en ce qui concerne les personnes vulnérables ou défavorisées et les violations des droits de la personne;

- en élaborant des mémoires et déclarations de principes à propos de la politique, des programmes gouvernementaux et des lois;

- en faisant la promotion du Code de déontologie de la profession.

L'ATTSO offre aux membres toute une gamme de services personnels et professionnels, notamment:

- une assurance responsabilité professionnelle globale à un prix fortement réduit;

- une ligne gratuite pour obtenir des conseils juridiques préliminaires;

- l'accès à des services axés sur Internet comme les groupes d'intérêts professionnels;

- un abonnement gratuit au Bulletin de l'ATTSO, au Bulletin de l'ACTS et au Travail social canadien; 
- une assurance domicile et automobile, des locations de voitures et de camions, des services de communications sans fil, des services financiers, etc., à prix réduit;

- des prix réduits pour les livres, les publications sur la profession ou l'emploi etc.;

- du matériel de recherche d'emploi et un bulletin d'emploi régulier, offerts gratuitement;

- une trousse d'informations pour «lancer » sa pratique privée;

- des taux préférentiels pour les programmes et conférences de formation continue.

La cotisation à l'ATTSO couvre également l'adhésion aux chapitres et sous-chapitres, ce qui donne la possibilité:

- de se pencher sur l'impact régional des politiques et programmes gouvernementaux;

- de jouer un rôle de leader en participant aux activités de conseils d'administration et de comités sur une diversité de questions sociales et professionnelles;

- d'établir des contacts avec des collègues à l'échelle locale;

- d'avoir accès à la formation continue et aux informations locales, par le biais, entre autres, des bulletins que publient la plupart des chapitres, etc.;

- de permettre aux étudiantes et étudiants en service social d'en savoir plus sur la profession.

Deux services très appréciés par les membres de l'ATTSO sont les informations sur la Semaine du service social et le Bulletin de l'ATTSO.

Chaque année, l'ATTSO célèbre la Semaine du service social la première semaine de mars (en 2002, du 4 au 10 mars). Vous pouvez obtenir de l'Association, des idées d'activités pour souligner la contribution des travailleuses et travailleurs sociaux ainsi que des matériaux publicitaires, entre autres, des affiches, des blocsnotes, des tasses, des marques-pages, des stylos et des ballons. Le but de la semaine est d'augmenter les informations au sujet du rôle des travailleuses et travailleurs sociaux et hausser le profil de la profession auprès de nos collègues et le public. 
En 2000-2001, le Bulletin de l'ATTSO a continué d'être un important véhicule de communication pour ce qui est des principales questions d'intérêt et préoccupations pour la profession. Les thèmes des derniers numéros ont été les suivants: «Les années 90, une décennie de changements - Où en est le service social?» (printemps 2000), "La pratique du service social: défis et possibilités» (automne 2000), «Leadership et innovation» (hiver 2001) et «Les droits de la personne» (été 2001).

Durant la dernière année, l'ATTSO a continué de jouer un rôle important sur le plan de l'intervention sociale et professionnelle. Nous aimerions montrer un aperçu de quelques-unes des initiatives.

- À la suite d'une conférence de presse tenue en octobre 2000 (pour coïncider avec la Marche mondiale des femmes) qui annonçait cet événement à venir, un forum communautaire d'un jour sur le thème, "Trouver des solutions à la violence envers les femmes et les enfants», a eu lieu à Toronto en mai 2001 et a remporté un vif succès. L'ATTSO avait mené cette initiative dont l'objectif était de rassembler des partenaires communautaires de divers secteurs des services sociaux ainsi que des conseillers en politiques des ministères gouvernementaux pertinents, afin d'entamer un dialogue sur cette question et d'élaborer des plans d'action. Cet événement a été financé par la Direction générale de la condition féminine de l'Ontario.

Andrée Côté de l'Association nationale de la femme et du droit était la principale conférencière. Mme Côté, qui possède une vaste expérience en droit criminel et droit de la famille ainsi que sur la question de l'égalité des droits des femmes victimes de violence, a fourni le contexte aux délibérations de cette journée. Un groupe de quatre experts dans le domaine de la violence envers les femmes et les enfants ainsi qu'une survivante, ont présenté leurs diverses perspectives sur la question. Près de cent (100) personnes ont participé à des discussions en petits groupes qui ont donné lieu à des recommandations pour des stratégies et orientations futures. 
L'ATTSO a préparé et distribué par courriel les résultats préliminaires du forum et s'est engagée à mettre au point un compte rendu du forum.

- En janvier 2001, l'ATTSO a envoyé une lettre à John Baird, ministre des Services sociaux et communautaires, afin d'exprimer notre appui sans réserve au gouvernement provincial pour son initiative visant à financer une ligne d'aide pour les femmes victimes de violence dans toute la province. Nous avons indiqué que la ligne d'aide actuelle pour les femmes victimes de violence n'était valable que pourToronto et qu'il existait d'importantes données étayant le besoin d'un financement accru pour couvrir les lacunes locales dans les services, pour avoir une ligne ATS/ATM pour les personnes sourdes et malentendantes et pour répondre aux besoins des femmes handicapées, des lesbiennes, des femmes des régions rurales du Nord, des immigrantes et des femmes de couleur.

- L'ATTSO a continué à être un partenaire provincial actif de Campagne 2000 (établie en 1989 en tant que coalition nationale d'organismes voués à l'élimination de la pauvreté chez les enfants canadiens d'ici l'an 2000).

- En novembre 2000, les coprésidentes du comité sur les questions relatives au vieillissement ont fait une présentation à la Commission ontarienne des droits de la personne (CODP). Dans des exposés oraux et des articles, l'ATTSO a répondu aux questions soulevées dans le document de consultation de la CODP sur le vieillissement et la discrimination, intitulé $L e$ visage changeant de l'Ontario: la discrimination et notre population vieillissante (2000). La réponse de l'Association portait sur un certain nombre de domaines où les aînés sont traités inégalement et on soulignait en particulier l'expérience des femmes d'un certain âge. La présentation de l'ATTSO a reçu un accueil favorable. En fait, dans le numéro de février 2001 du magazine Fifty Plus de CARPNews (Association canadienne des individus retraités), l'article de fond est consacré à Keith C. Norton, commissaire ontarien des droits de la personne qui cite les commentaires de l'ATTSO sur l'âgisme ou la discrimination fondée sur l'âge. 
- L'ATTSO a continué à participer activement à une alliance interdisciplinaire composée de cinq associations professionnelles (diététistes et nutritionnistes, ergothérapeutes, physiothérapeutes, orthophonistes et audiologistes) sous le nom de «Alliance of Professional Associations for Community-Based Therapy Services» (Alliance d'associations professionnelles pour les services de thérapie communautaires (APACTS). Le mandat de l'APACTS consiste à examiner la manière dont les associations peuvent collaborer pour faire face à leurs préoccupations communes au sujet de l'érosion potentielle des normes et la marginalisation des professionnels qui fournissent des services aux Centres d'accès aux soins communautaires (CASC). Les initiatives entreprises actuellement par l'APACTS consistent entre autres : à mettre au point une trousse de documents qui sera distribuée à tous les CASC, vers le début juin, pour promouvoir une meilleure utilisation des services de thérapie au sein du secteur des soins à domicile (chacune des professions a préparé pour cette trousse une page recto-verso dans laquelle elle décrit son rôle et les services qu'elle offre - cette documentation s'adresse aux gestionnaires de cas au sein des CASC); et à envoyer une lettre aux dirigeants des CASC de toute la province pour promouvoir les qualifications de nos membres et encourager les CASC à recruter et embaucher des membres de nos professions aux postes de gestionnaires de cas, occupés à présent presque essentiellement par des infirmiers ou infirmières.

- L'ATTSO participe à un projet de recherche conjoint avec Judy Globerman, Ph.D., Faculté de service social de l'Université de Toronto, et Jane White, coordonnatrice de stages à l'École de service social de la Niagara University (Buffalo, USA), sur les questions émergentes concernant les répercussions de la restructuration des hôpitaux sur la pratique du service social. Ce projet permettra d'identifier les stratégies auxquelles ont recours les travailleurs sociaux pour gérer leurs nouveaux milieux de travail. Les résultats de cette recherche seront présentés dans le Bulletin de l'ATTSO et dans une publication spéciale. 
- En avril 2001, le comité du service social scolaire a publié le $18^{\text {e }}$ numéro de son bulletin qui est distribué à 400 travailleurs sociaux scolaires, non seulement dans tout l'Ontario mais également à l'échelle internationale à de nombreux États américains et à 12 pays différents.

- Le symposium du service social scolaire 2001, qui a eu lieu le 26 octobre 2001, était co-parrainé par le conseil scolaire du district catholique de London et le conseil scolaire du district de Thames Valley et avait pour thème: «L'école en pleine tourmente - prestation d'un continuum de services attentifs en ces temps difficiles». Le conférencier principal était Michel Lespérance.

- Le comité du service social scolaire met continuellement à jour un sondage sur le service social scolaire au sujet des travailleurs sociaux scolaires et de leur milieu de travail au sein des conseils scolaires dans tout l'Ontario. Ce sondage couvre différentes données comme les titres d'emploi, les qualifications, la gestion et l'obligation de rendre compte de sa pratique, les effets des compressions budgétaires, les charges de cas, les conditions d'emploi, etc.

- Le 18 janvier 2001, l'ATTSO a rencontré des responsables de la Division Ontario au travail du ministère des Services sociaux et communautaires (MSSC) au sujet de la proposition du gouvernement concernant le traitement obligatoire pour toxicomanie auquel devront se soumettre les assistés sociaux. L'Association a pris part à un processus de consultation et cherché à mettre à jour et clarifier cette proposition gouvernementale. Nous avons également souligné les préoccupations de la profession au sujet de questions comme la stigmatisation des assistés sociaux, le recours à un traitement obligatoire et la violation des droits de la personne.

- L'ATTSO a continué à défendre les travailleurs sociaux pour qu'ils figurent sur la liste des professions pouvant remplir le formulaire sur les activités de la vie quotidienne, destiné aux personnes qui cherchent à obtenir des prestations d'invalidité. Notre première correspondance avec le gouvernement à ce sujet remonte au mois de septembre 1998. En décembre 2000 
et janvier 2001, l'Association a réitéré sa préoccupation au sujet de l'omission des travailleurs sociaux de la liste des professions habilitées à remplir et signer ce formulaire et de l'exclusion des travailleurs sociaux du processus de consultation gouvernementale qui a eu lieu l'automne dernier. En septembre 2001, une rencontre a eu lieu avec le sous-ministre des Services sociaux et communautaires.

- L'ATTSO a appuyé et participé à la campagne «Appelez Mike» du comité relatif aux lois sur les invalidités en Ontario. La campagne invitait les partisans, leurs familles et amis à bombarder le bureau de Mike Harris d'appels téléphoniques réclamant que le Premier ministre tienne la promesse de sa campagne de 1995 visant à adopter des lois fortes, efficaces et obligatoires en faveur des Ontariens et Ontariennes invalides. Des informations sur cette campagne ont été affichées sur le site Web de l'ATTSO, de la documentation a été envoyée à nos chapitres les encourageant à organiser des groupes pour faire des appels téléphoniques et, des informations ont été envoyées dans les trousses de renouvellement de l'adhésion et avec les accusés de réception de septembre 2000 à avril 2001.

L'ATTSO a aussi un groupe assidu et dévoué qui forme le comité francophone provincial. Cette année, le comité s'est consacré à l'élaboration d'un document de recommandations pour le Conseil d'administration pour soutenir une plus grande intégration du bilinguisme au sein de l'Association. Le comité a aussi contribué à l'assemblée annuelle 2000 de RIFSSSO en présentant une demi-journée de formation consacrée aux «Thérapies alternatives» et un Forum de discussion portant sur «L'action sociale».

En conclusion, les membres de l'ATTSO représentent toute la pratique du service social. Si vous êtes diplômé d'un programme universitaire en service social accrédité au niveau du baccalauréat (BSS), de la maitrise (MSS) ou du doctorat (Ph. D.) ou étudiant dans l'un de ces programmes et si vous résidez, étudiez ou travaillez en Ontario, vous pouvez devenir membres de l'ATTSO. 
Pour plus d'informations, visitez notre site Web www.oasw.org ou composez le 416-923-4848. Joignez votre voix à celle de votre profession et tirez parti des avantages de l'adhésion à l'Association des travailleuses et travailleurs sociaux de l'Ontario. 